\title{
Fatal disseminated cryptococcosis following intraocular involvement
}

\author{
JOEL A SCHULMAN,' CHRISTOPHER LEVEQUE,'2 MARION COATS,' \\ LINDA LAWRENCE,' AND JOHN C BARBER'
}

From the Departments of 'Ophthalmology and ${ }^{2}$ Pathology, University of Texas Medical Branch, Galveston, Texas 77550, USA

SUMMARY A 33-year-old man was treated with systemic steroids for a retinal inflammatory lesion before the diagnosis of cryptococcal retinitis and meningitis was suspected. He died from central nervous system disease despite treatment with parenteral antifungals. Histopathological studies demonstrated ocular and disseminated systemic infection with Cryptococcus neoformans. Direct cryptococcal involvement of the eye is rare and is usually associated with disseminated disease. Systemic steroids must be used with caution, and patients who take these drugs require frequent monitoring.

Cryptococcosis is a mycosis caused by a non-mycelial yeast-like fungus, Cryptococcus neoformans. The organism is worldwide in distribution and endemic in the human population in the Mississippi Valley. The primary lesion is usually in the lung, with dissemination most frequently to the meninges and brain or spinal cord. Other reported sites of cryptococcus include the skin, bones, and liver. ${ }^{12}$ Direct cryptococcal involvement of the eye is rare and usually associated with disseminated disease. ${ }^{3}$

We present a patient treated with systemic steroids for a non-pigmented retinal inflammatory lesion before the diagnosis of cryptococcal retinitis and meningitis was established. The patient died from the central nervous system disease despite treatment with parenteral antifungals.

\section{Case report}

The patient was a 33-year-old male in good health until September 1984, when he developed decreased vision, pain, and photophobia in his left eye. The patient lived in a wooded area where he raised dogs as a hobby, and was exposed to pigeons. Two months prior to ophthalmological examination he reported accidental eye contact with parvo and leptospirosis vaccine while inoculating his dogs. His past medical history included the consumption of one pint to one quart $(600-1200 \mathrm{ml})$ of spirits per day over the

Correspondence to Joel A Schulman, MD, 2849 Dominique, Galveston, Texas 77550, USA. previous year. He admitted to homosexual activity which stopped three years before initial presentation to an ophthalmologist. He denied parenteral use of illicit drugs.

He was first examined by a local ophthalmologist on 11 January 1984, at which time his best corrected visual acuity was $20 / 20$ in the right eye and $20 / 40$ in the left eye. The right eye was completely normal. The left eye had a cellular reaction in both the anterior chamber and vitreous cavity. A slightly elevated yellowish retinal lesion approximately $3 \frac{1 / 2}{1}$ by 5 disc diameters in size involving the temporal macula (Fig. 1) was observed. The patient was diagnosed as having an unspecified infectious chorioretinitis. Homatropine $5 \%$ four times daily and prednisolone acetate $1 \%$ every two hours were prescribed for the left eye.

Tests for acquired immune deficiency disorder were negative. Additional test results included a negative rapid plasmin reagent test for syphilis (RPR) and a negative hepatitis B surface antigen. A test of leptospira titre was negative; histoplasmosis (mycelial) and histoplasmosis (yeast) titres were both less than 1:8 and toxoplasmosis titre was less than $1: 16$. The cytomegalovirus titre was $1: 128$ and the complete blood cell count and differential were unremarkable except for a mildly increased white blood cell count of $9 \cdot 4 \times 10^{4} / 1$.

Re-examination on 7 March showed that the patient could barely count fingers with the left eye. The vitreous contained a large number of non- 


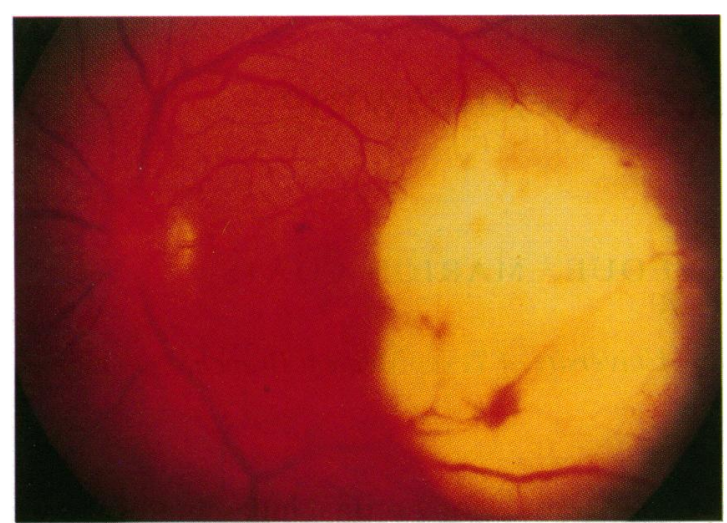

Fig. 1 Fundus photograph of the left eye showing a slightly elevated retinal lesion involving the temporal portion of the macula and inferior arcade. Retinal haemorrhages are present over the lesion. The overlying retinal vessels appear to be involved in the inflammatory process.

pigmented cells, and the retinal lesion had enlarged (Fig. 2). Oral prednisone $100 \mathrm{mg}$ daily was initiated and tapered to $60 \mathrm{mg}$ after three days. Best corrected vision in the left eye one week later improved to 20/200 and the patient reported decreased ocular pain. On 21 March the fundus lesion was smaller, and the oral prednisone was decreased to $40 \mathrm{mg}$ daily.

The patient went to an emergency room on 6 April 1984 with a six-day history of fever up to $39 \cdot 4^{\circ} \mathrm{C}$, nausea (without vomiting), anorexia, confusion, neck stiffness, headaches in the occipital and nuchal region, and photophobia and blurred vision of the left eye. The oral prednisone had been tapered to

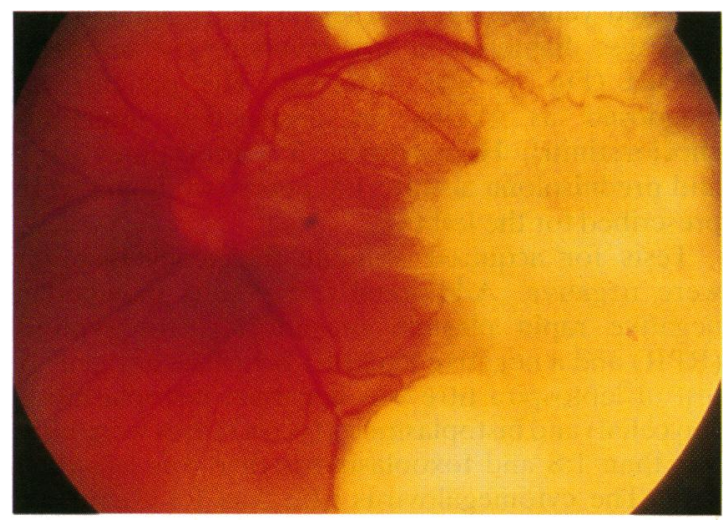

Fig. 2 Left eye approximately two months later. The lesion has enlarged and now extends beyond the temporal macula. The inferior aspect appears lobulated. A preretinal membrane extends from the retinal lesion to the optic disc. Retinal vessels appear to be constricted and disappear within the lesion.
$20 \mathrm{mg}$ daily. The patient was transferred to the University of Texas Medical Branch (UTMB) at Galveston after receiving intravenous fluids for dehydration.

When admitted to UTMB, Galveston, the patient's temperature was $39 \cdot 5^{\circ} \mathrm{C}$, blood pressure $136 / 80 \mathrm{mmHg}$, pulse $100 / \mathrm{min}$, and respiration $20 /$ min. The patient appeared lethargic and confused. Physical examination was normal except for a few enlarged cervical lymph nodes, inappropriate affect, and execution of commands in an opposite manner to the directive about $50 \%$ of the time.

Visual acuity in the right eye was 20/20 and findings were normal. In the left eye the visual acuity was $20 / 400$, and examination revealed numerous vitreous cells and a yellowish retinal lesion temporal to the optic disc associated with an overlying vitreous infiltrate.

The opening pressure on lumbar puncture was 333 $\mathrm{mm} \mathrm{H}_{2} \mathrm{O}$ with a closing pressure of $160 \mathrm{~mm} \mathrm{H}_{2} \mathrm{O}$. An India ink preparation of cerebrospinal fluid (CSF) demonstrated organisms resembling Cryptococcus neoformans. The cryptococcal latex agglutination test was positive for cryptococcal antigen in a dilution of 1:64 when performed on both blood and CSF. Cultures of CSF failed to isolate any organisms.

A diagnosis of cryptococcal meningitis, retinitis, and possible endophthalmitis was made, and the patient was started on intravenous amphotericin B and oral 5-flucytosine. His neurological status deteriorated despite therapy. A third nerve palsy developed on the second day of admission and he developed apnoeic spells four days later. A CT scan the following day showed posterior fossa infarcts and hydrocephalus.

Four days later a right frontal indwelling ventricular reservoir system (Omaya reservoir) for intrathecal amphotericin B administration was placed. The patient continued on a downhill course and required intubation and tracheostomy. He became comatose and died on day 30 in hospital.

PATHOLOGICAL EXAMINATION

The necropsy showed disseminated cryptococcosis with cryptococcal granulomata present in the central nervous system, liver, adrenal glands, and both kidneys. Microscopic examination of the left eye revealed numerous cryptococcal organisms infiltrating the retina (Fig. 3). Choroidal and optic nerve involvement was not observed.

\section{Discussion}

Cryptococcosis is caused by Cryptococcus neoformans, an encapsulated saprophytic yeast-like fungus which grows best at $37^{\circ} \mathrm{C}$. The organism is 


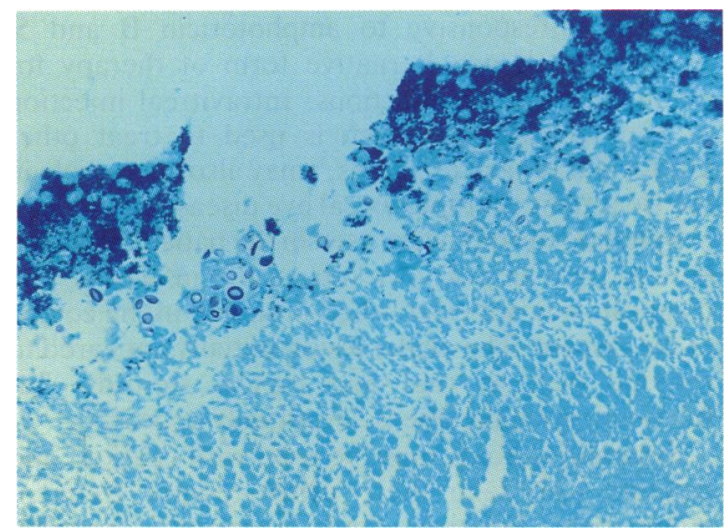

Fig. 3 Tangential microscopic section (left eye) through the retina revealing a cluster of cryptococcal organisms within the layer of rods and cones. The pigment epithelium is attached. Gomorimethenamine silver.

found in high concentrations in soil that contains pigeon faeces, though the birds are not naturally infected. The organism has also been recovered from various animals (including dogs, cats, and cows), fresh vegetable skins, and fruits. ${ }^{124}$

Human infection is acquired by inhalation of contaminated particles. The primary pulmonary infection may be asymptomatic but be followed by haematogenous spread to other areas of the body. ${ }^{2}$ The organism has a predilection for the CNS. Direct animal-to-man transmission has not been documented,' and only one case of direct person-toperson transmission has been reported. A case of exogenous intraocular cryptococcal endophthalmitis followed corneal transplantation with donor material from a patient with disseminated cryptococcosis. ${ }^{5}$ New cases of the disease are rare, approximately 300 being reported annually in the United States.' This suggests a strong natural resistance to the organism. Although $50 \%$ of the cases of cryptococcosis occur in otherwise healthy individuals, a three-fold increase of this infection exists among patients with sarcoidosis, lymphoreticular malignancies, or steroidal immunosuppression. ${ }^{14}$ Cryptococcosis also occurs more frequently in patients with acquired immune deficiency' or diabetes. $^{1+}$ Therapy with steroids or immunosuppressives appears to increase individual susceptibility to cryptococcosis and dissemination of dormant primary lesions. ${ }^{1+}$ Cirrhosis of the liver is associated with an extremely fulminant form of the disease. $^{2}$

Cryptococcus neoformans is the most common fungus causing CNS infections, usually meningoencephalitis or meningitis. In rare instances patients with CNS involvement may be afebrile and display no neurological signs or symptoms. ${ }^{6}$
Cryptococcus neoformans rarely causes primary ocular disease. The organism may invade the eye either as a result of direct extension along the optic nerve or its meningeal sheath or by haematogenous dissemination. ${ }^{3}$ Histological studies show that endogenous ocular cryptococcosis is primarily a choroidal disease, with secondary invasion of the sensory retina and other intraocular structures. ${ }^{7}$. Cryptococcus neoforms has also been reported to cause endophthalmitis, uveitis, and retinitis. ${ }^{3}$ The histological reaction caused by the organism in the choroid and retina can range from minimal to no inflammatory reaction or necrosis to granulomatous changes. ${ }^{\text {? }}$

Secondary involvement of the eye as a consequence of CNS involvement is more common. Approximately $40 \%$ of individuals with cryptococcal meningitis have eye signs or symptoms. Papilloedema, optic atrophy, and extraocular muscle paresis were the most common ophthalmic signs in a series of 36 cases of cryptococcal meningitis reported by Okun and Butler. ${ }^{8}$ Decreased visual acuity with CNS involvement appears in many patients to be caused by direct invasion of the visual pathways by the cryptococcal organism. ${ }^{8}$

The differential diagnosis of a posterior, slightly elevated, amelanotic choroidal, retinal, or chorioretinal lesion includes melanoma, metastatic carcinoma, or other neoplastic ocular diseases such as leukaemia, haemangioma, toxocariasis, osteoma, chorioretinal fungal abscess, cryptococcal chorioretinitis or retinitis, and serous or disciform extramacular or macular detachment. Syphilis and tuberculosis must also be ruled out.

Several other infectious agents including cytomegalovirus, Candida albicans, Toxoplasma gondii, and herpes hominis virus usually present as a retinitis with varying degrees of secondary choroidal involvement. Affected patients have areas of opacified retina with overlying vitreous cells. In contrast the initial lesion in cryptococcal chorioretinitis develops in the choroid with secondary retinal involvement. ${ }^{79}$ Vitreous overlying the chorioretinal lesions usually remains clear. ${ }^{6}$

Funduscopic examination by indirect ophthalmoscopy, a fundus contact lens examination, and the use of adjunct diagnostic tests including quantitative ultrasonography and fluorescein angiography are useful in differentiating along the several conditions presenting as moderately elevated posterior chorioretinal or retinal masses.

Special serological tests such as detection of IgG antibodies against Toxoplasma gondii ${ }^{10}$ or the ELISA test (enzyme-linked immunosorbent assay) in suspected ocular toxocariasis" may help confirm a suspected diagnosis. A clinical diagnosis of a retinitis caused by herpes hominis or cytomegalovirus may be 
supported by demonstrating rising serum antibody titres to the virus or recovery of the virus from blood, urine, aqueous, or the throat. ${ }^{9}$

The diagnosis in a patient with a suspected cryptococcal chorioretinal or retinal lesion is complicated by the frequent association between direct intraocular cryptococcal involvement and the disseminated form of the infection, which frequently involves the central nervous system. ${ }^{12}$ Routine laboratory tests such as complete blood count and sedimentation rate usually give normal results. Identification of the encapsulated organism in cerebrospinal fluid stained with India ink is sufficient for making a presumptive diagnosis and initiating treatment pending culture results. ${ }^{4}$ India ink preparations are negative in $50 \%$ of cases involving the central nervous system. 'In suspected cases the urine, CSF, blood, and sputum should be cultured even in the absence of evidence suggesting genitourinary or pulmonary infection.

Cryptococcus neoformans grows well on both blood agar and Sabouraud's medium. ${ }^{13}$ Cycloheximide, which is added to most fungal media to inhibit non-pathogens, also inhibits $C$. neoformans and should not be present in the agar.' Several serological tests for cryptococcus have been developed including complement fixation, tube agglutination, immunodiffusion, and an indirect immunofluorescence test. ${ }^{14}$ The latex agglutination test for detection of antigen on the cryptococcal polysaccharide capsule is the most commonly used commercially available test. A titre greater than $1: 8$ is an indication for treatment. ${ }^{8}$ The presence of antigen should be tested in blood, urine, and CSF if the diagnosis is suspected but not proved.

When tests of serum, sputum, CSF, and blood are negative and a high index of suspicion still exists, a diagnostic vitreous tap, ${ }^{9}$ vitrectomy, ${ }^{7}$ or eye wall biopsy ${ }^{15}$ can be performed to obtain tissue specimens suitable for histological examination and culture. Morphological characteristics of the organism which allow identification are apparent with periodic acid Schiff (PAS) or methenamine silver stains. Mayer's mucicarmine will stain the polysaccharide capsule red, which differentiates Cryptococcus neoformans from other organisms and artefacts.'

Before the introduction of the antifungal agent amphotericin B disseminated cryptococcal infections were invariably fatal, and most cases with direct ocular involvement were positively diagnosed either at necropsy or following enucleation. ${ }^{12}$ Parenteral amphotericin B alone $e^{8914}$ or in combination with 5 -flucytosine ${ }^{39}$ has been used successfully in the treatment of some cases of intraocular cryptococcosis. Miconazole, which has been reported to be effective in a case of central nervous system crypto- coccosis unresponsive to amphotericin B and 5flucytosine,$^{3}$ is an alternative form of therapy for myotic intraocular infections. Intravitreal injection of amphotericin B, which is used to treat other intraocular fungal infections, ${ }^{16}$ may also have a role in the treatment of cryptococcal eye disease.

Systemic steroids were used in the patient reported here to treat an unknown intraocular infection. The diagnosis was not established until late in the course of the disease. The ocular lesion initially appeared to respond well to oral and topical steroids, but fulminant systemic disease eventually appeared.

Systemic steroids must be used with caution, and patients who take these drugs require frequent monitoring. Fujikawa and associates ${ }^{17}$ reported hyperosmolar hyperglycaemic non-ketotic coma in three patients who received systemic steroid therapy for ocular disorders. Two of these patients died.

This case demonstrates the diagnostic difficulties that may be encountered in a patient presenting with an apparently inflammatory retinal lesion with overlying vitreous involvement. Cryptococcus neoformans must be considered in the assessment of such a lesion, or a delay in diagnosis and treatment may occur.

\section{References}

1 Diamond RD. Cryptococcus neoformans. In: Mandell GL, Douglas RG Jr, Bennett JE, eds. Principles and practice of infectious diseases. 2nd ed. New York: Wiley, 1979: 1460-8.

2 Myerowitz RL. The pathology of opportunistic infections with pathogenetic, diagnostic, and clinical correlations. New York: Raven Press, 1983: 145-60.

3 Bisseru B, Bajaj A, Carruthers RH, Chhabbra HN. Pulmonary and bilateral retinochoroidal cryptococcosis. Br J Ophthalmol 1983; 67: 157-61.

4 Littman ML, Walter JE. Cryptococcosis: current status. Am J Med 1968; 45: 922-32.

5 Beyt BF, Waltman Sr. Cryptococcal endophthalmitis after corneal transplantation. $N$ Engl J Med 1978; 298: 825-6.

6 Salaki JS, Louria DB, Chmel H. Fungal and yeast infections of the central nervous system. Medicine 1984; 63: 108-32.

7 Shields JA, Wright DM, Augsburger JJ, Wolkowicz MI. Cryptococcal chorioretinitis. Am J Ophthalmol 1980; 89: 210-8.

8 Okun E, Butler WT. Ophthalmologic complications of cryptococcal meningitis. Arch Ophthalmol 1964; 71: 52-7.

9 Doft BH, Curtin VT. Combined ocular infection with cytomegalovirus and cryptococcosis. Arch Ophthalmol 1982; 100: 1800-3.

10 Rothova A, van Knapen F, Baarsma GS, Krait PJ, LoewerSieger DH, Kijlstra A. Serology in ocular toxoplasmosis. $\mathrm{Br} J$ Ophthalmol 1986; 70: 615-22.

11 Pollard ZF, Jarrett WH, Hagler WS, Allain DS, Schantz PM. ELISA for diagnosis of ocular toxocariasis. Ophthalmology 1979; 86: 743-9.

12 Cameron ME, Harrison A. Ocular cryptococcosis in Australia, with a report of two further cases. Med J Aust 1970; i: 935-8.

13 Armstrong D. Fungal infections in the compromised host. In: Rubin RH, Young. LS, eds. Clinical approach to infection in the compromised host. New York: Plenum, 1981: 219-20. 
14 Wolf PL. Immunofluorescence as a diagnostic aid in cryptococcal meningitis and other fungal infections. Am J Pathol 1975; 78: $17 \mathrm{a}$.

15 Peyman GA, Juarez CP, Raichand M. Full-thickness eye-wall biopsy: long-term results in 9 patients. Br J Ophthalmol 1981; 65: 723-6.
16 Perraut LE Jr, Perraut LE, Bleiman B, Lyons J. Successful treatment of Candida albicans endophthalmitis with intravitreal amphotericin B. Arch Ophthalmol 1981; 99: 1565-7.

17 Fujikawa LS, Meisler DM, Nozik RA. Hyperosmolar hyperglycemic nonketotic coma: a complication of short-term systemic corticosteroid use. Ophthalmology 1983; 90: 1239-41.

Accepted for publication 22 December 1986. 